hep-ph/9804392

Aug 31, 1998

\title{
Unstable Higgs Particle and the Equivalence Theorem
}

\author{
R.S. Willey ${ }^{a}$ \\ ${ }^{a}$ Department of Physics and Astronomy \\ University of Pittsburgh, Pittsburgh, PA 15260, USA
}

\begin{abstract}
We consider the subtleties involved in the application of the Equivalence Theorem to the decay of an unstable Higgs particle. This is formally justified from consideration of unitarity and resonant elastic scattering of the stable decay prodiucts. By explicit perturbative calculation we find that the imaginary parts of the one-loop amplitudes for that decay are in agreement with the Equivalence Theorem only when one includes the imaginary parts generated by evaluating the tree-level amplitudes at the complex on-shell value of the four-momentum squared of the unstable particle.
\end{abstract}

\footnotetext{
${ }^{1}$ E-mail: willey@vms.cis.pitt.edu
} 


\section{Introduction}

The Equivalence Theorem is a property of gauge theories with Spontaneous Symmetry Breaking. The gauge vector bosons acquire mass via the vev of a scalar (Higgs) field. In a $R_{\xi}$ gauge there are also one or more unphysical wouldbe Goldstone bosons. The Equivalence Theorem asserts that, in the limit in which the energies are all much greater than the vector boson masses, S-Matrix elements with external longitudinal polarized gauge vector bosons are equal to (up to constant overall phase) S-Matrix elements which have the external longitudinal polarized vector bosons replaced by external wouldbe Goldstone bosons of the same four-momenta. For Green Functions, the statement is true at tree level, but loop corrections may introduce renormalization scheme dependent correction factors. There is a substantial literature on this subject which may be traced from the recent review by He,Kuang, Yuan [1].

The derivations all rely, explicitly or implicitly, on the LSZ reduction formulas to relate perturbatively computed renormalized $\tau-$ functions (Green Functions) to S-Matrix elements. This in turn implies that external lines in Feynman diagrams (for S-Matrix elements ) represent stable particles in asymptotic states (physically, sufficiently long lived to reach detectors outside the interaction region). One may then ask if the Equivalence Theorem can be applied to the decay of an unstable Higgs boson that is known only by the detection of its stable decay products. Is the invariant matrix-element for the decay of a heavy Higgs particle into two longitudinal polarized gauge bosons the same as that for the decay of the Higgs particle into two wouldbe Goldstone bosons?

The simplest model in which one can study these matters is the Abelian Higgs model. In section two we go over the renormalization and BRST structure of this model. In section three we determine the finite ratios of field strength renormalization constant required for LSZ to relate renormalized $\tau-$ functions to S-Matrix elements when all the particles are stable. In section four we take up the questions raised by the instablity of the Higgs boson. We give a heueristic continuity argument and then a derivation based on unitarity of the resonance contribution to the scattering of stable particles that formally justifies application of the Equivalence Theorem to this case. In section five we check the general results by explicit perturbative (tree plus one-loop) calculation in the Abelian Higgs model. In section six the corresponding perturbative calculations are done for the Standard Model of the electroweak interactions.

In both cases, we find that the simple imaginary parts of the one-loop amplitudes (which directly reflect the instablity) agree only after they are combined with the imaginary parts generated by evaluating the tree-level amplitudes at the complex on-shell value of the four-mommentum squared of the unstable particle.

\section{Abelian Higgs Model and BRST}

The 'classical' Lagrangian is

$$
\mathcal{L}_{c l}=-\frac{1}{4} A_{0_{\mu \nu}} A_{0}^{\mu \nu}+\left|D_{0} \phi_{0}\right|^{2}-\mu_{0}^{2}\left|\phi_{0}\right|^{2}-\lambda_{0}\left(\left|\phi_{0}\right|^{2}\right)^{2}
$$

where

$$
A_{0}^{\mu \nu}=\partial^{\mu} A_{0}^{\nu}-\partial^{\nu} A_{0}^{\mu}, \quad D_{0}^{\mu} \phi_{0}=\left(\partial^{\mu}+i g_{0} A_{0}^{\mu}\right) \frac{1}{\sqrt{2}}\left(h_{0}-i \chi_{0}\right)
$$

The subscript zero denotes canonical unrenormalized fields and bare parameters. 
Perturbatively, for $\mu_{0}^{2}>0$, the system is in the symmetric phase with $<0\left|h_{0}\right| 0>=$ 0 . For $\mu_{0}^{2}<0$, the system is in spontaneously broken symmetry phase with

$$
<0\left|\phi_{0}\right| 0>=\frac{1}{\sqrt{2}}<0\left|h_{0}\right| 0>=\frac{1}{\sqrt{2}} V_{0} .
$$

we separate $h_{0}=V_{0}+\hat{h}_{0}$.

We choose a gauge fixing function of the Feynman-t'Hooft class to cancel the tree level $\chi-\partial A$ mixing generated in (11) by the above field shift in the broken symmetry phase .

$$
\begin{gathered}
\mathcal{L}_{G F}=-\frac{1}{2 \xi_{0}} F_{0}^{2} \\
F_{0}=\partial A_{0}+\xi_{0} g_{0} \kappa_{0} V_{0} \chi_{0}
\end{gathered}
$$

The second gauge parameter $\kappa_{0}$ is required for consistent renormalization of $F_{0}$. (See below ). With this gauge fixing the Fadeev-Popov effective Lagrangian is

$$
\begin{aligned}
\mathcal{L}_{F P} & =\text { const } \bar{c}_{0} \frac{\delta F_{0}}{\delta \omega} c_{0} \\
& =\bar{c}_{0}\left(-\partial^{2}-\xi_{0} g_{0}^{2} \kappa_{0} V_{0}\left(V_{0}+\hat{h}_{0}\right)\right) c_{0}
\end{aligned}
$$

The BRST transformations under which $\mathcal{L}_{c l}$ and $\mathcal{L}_{G F}+\mathcal{L}_{F P}$ are invariant are:

$$
\begin{array}{cl}
\delta_{0} A_{0}^{\mu}=-\partial^{\mu} c_{0}, \quad \delta_{0} \hat{h}_{0}=g_{0} \chi_{0} c_{0} & \delta_{0} \chi_{0}=-g_{0}\left(V_{0}+\hat{h}_{0}\right) c_{0} \\
\delta_{0} c_{0}=0, & \delta_{0} \bar{c}_{0}=-\frac{1}{\xi_{0}} F_{0}, \quad \delta_{0} F_{0}=0
\end{array}
$$

We now reparametrize the Lagrangian in terms of renormalized fields and parameters. We require that this be done in such a way that the renormalized fields and parameters satisfy the same BRST relations (6) and Ward identites as the unrenormalized fields and parameters.

$$
\begin{array}{rrr}
A_{0}=\sqrt{Z_{A}} A, & \left(\hat{h}_{0}, \chi_{0}, V_{0}\right)=\sqrt{Z}(\hat{h}, \chi, V) & \\
\mu_{0}^{2}=Z_{\mu^{2}} \mu^{2}, & \lambda_{0}=Z_{\lambda} \lambda, & g_{0}=Z_{g} g \\
\xi_{0}=Z_{\xi} \xi, & \kappa_{0}=Z_{\kappa} \kappa & \\
c_{0}=\sqrt{Z_{c}} c, & \bar{c}_{0}=\sqrt{Z_{\bar{c}}} \bar{c} &
\end{array}
$$

The vertex renormalization factors are:

$$
Z_{4 \lambda}=Z^{2} Z_{\lambda}, \quad Z_{3 g}=Z \sqrt{Z_{A}} Z_{g}, \quad Z_{4 g}=Z Z_{A} Z_{g}^{2}
$$

The Abelian Ward identities provide the relations

$$
Z_{\xi}=Z_{A}, \quad Z_{3 g}=Z\left(Z_{g}=Z_{A}^{-\frac{1}{2}}\right), \quad Z_{4 g}=Z
$$


With these definitions and Ward identities, the BRST transformations of $A_{0}, \hat{h}_{0}, \chi_{0}$ all renormalize as

$$
\delta_{0} X_{0}=Y_{0}, \quad \delta_{0} X=\sqrt{\frac{Z_{c}}{Z_{A}}} Y
$$

So define renormalized BRST variation

$$
\delta_{0}=\sqrt{\frac{Z_{c}}{Z_{A}}} \delta
$$

The transformation of $c_{0}$ renormalizes trivially. For the transformation relating $c_{0}$ to $F_{0}$ we have

$$
F_{0}=\partial A_{0}+\xi_{0} g_{0} \kappa_{0} V_{0} \chi_{0}=\sqrt{Z_{A}} \partial A+Z_{\xi} Z_{g} Z_{\kappa} Z \xi g \kappa V \chi
$$

By the Ward identities, $Z_{\xi} Z_{g}=\sqrt{Z_{A}}$, so $Z_{\kappa} Z$ must be finite. Any finite residual can be absorbed in the as yet unspecified constant $\kappa$. So we take

$$
Z_{\kappa}=Z^{-1}
$$

Then

$$
F_{0}=\sqrt{Z_{A}}(\partial A+\xi g \kappa V \chi)=\sqrt{Z_{A}} F
$$

The last BRST transformation is then

$$
\delta_{0} \bar{c}_{0}=-\sqrt{\frac{Z_{c} Z_{\bar{c}}}{Z_{A}}} \delta \bar{c}=-\frac{1}{\sqrt{Z_{A}}} \frac{F}{\xi}
$$

which fixes

$$
Z_{c} Z_{\bar{c}}=1
$$

With these specifications, the renormalizations (7) preserve the structure of the BRST transformations (6). So the renormalized Green functions satisfy the same Ward identities and BRST relations as the (dimensionaly regularized) unrenormalized Green functions .

The gauge parameter $\xi$ will be left free, so the analysis will be for any $R_{\xi}$ gauge. The second gauge parameter will be fixed by the considerations given now. First $\kappa$ must be one at tree-level to cancel the tree-level $\chi-\partial A$ mixing. Next is the treatmment of the vev $\mathrm{V}$ and the vector boson mass $\mathrm{M}$. The exact renormalized vev $\mathrm{V}$ is determined order by order in perturbation theory, as a function of the renormalized parameters of the theory, by the requirement

$$
<0|\hat{h}| 0>=0
$$

that is, all tadpoles vanish. At tree-level,

$$
V^{0-\text { loop }} \equiv v=\sqrt{\frac{-\mu^{2}}{\lambda}}, \quad\left(\mu^{2}<0\right)
$$

Beyond tree-level $\mathrm{V}$ depends also on g, and particularly, on the gauge parammeter $\xi$. So the vector boson mass $\mathrm{M}$ is specified in terms of the gauge invariant tree-level vev.

$$
M=g v
$$


So we will take $\kappa$ to have a factor $\frac{v}{V}$. Finally, to make the Equivalence Theorem as a relation between S-matrix elements come out with no extra constant factors, we will include a factor $\sqrt{\frac{Z}{Z_{\chi}^{\star}}}$ in $\kappa$

$$
\kappa=\sqrt{\frac{Z}{Z_{\chi}^{\star}}} \frac{v}{V}
$$

Here, we distinguish between $Z$, the renormalization of $\chi_{0}$ introduced in (7) with no commitment as to the renormalization condition required to fix its value, and $Z_{\chi}^{\star}$ which is the renormalization of $\chi_{0}$ fixed by the renormalization condition that the pole of the complete renormalized $\chi$ two-point function have unit residue (derivative of the renormalized self-energy function vanishes at $\left.k^{2}=M_{\chi}^{2}\right)$. The field

$$
\chi^{\star}=\sqrt{\frac{Z}{Z_{\chi}^{\star}} \chi}
$$

is the renormalized field which satisfies the LSZ asymptotic condition with no finite field renormalization factor. With these specifications, the renormalized $\mathrm{F}$ is

$$
F=\partial A+\xi M \chi^{\star}
$$

Among the renormalization conditions necessary to complete the sppecification of the renormalization (17), we will always include the unit residue condition for the physical vector boson.

$$
A_{0}=\sqrt{Z_{A}^{\star}} A, \quad A=A^{*}
$$

The BRST analysis provides the result [2]

$$
<\beta, \text { phys }^{\prime}|F| \alpha, \text { phys }^{\prime}>=0
$$

Physical states may be taken to be in,out-states of physical particles whose asymptotic in,out-fields $(g \rightarrow 0)$ are BRST invariant. Since (6) has the $\chi$ field BRST invariant in the limit $g \rightarrow 0$, just as the $\hat{h}$ field, the states $\mid p h y s^{\prime}>$ in (23) may include wouldbe Goldstone bosons, as well as Higgs scalars and vector bosons in a physical polarization state (spacelike transverse or longitudinal in three dimensions, not timelike).

\section{The Equivalence Theorem}

The Equivalence Theorem is now a simple application of the LSZ reduction formulas, combined with(23). We will arrange renormalization conditions so that the masses in the renormalized free propagators are the physical masses (modulo problems posed by unstable particles). That is, renormalization constants are chosen so that renormalized self energy functions vanish on their respective mass shells.

$$
M_{A}^{2}=g^{2} v^{2} \equiv M^{2}, \quad m_{h}^{2}=2 \lambda v^{2} \equiv m^{2}, \quad M_{\chi}^{2}=\xi M^{2}
$$


We start with the LSZ reduction formula to take a single $\chi$ - particle out of an in-state.

$$
<\beta\left|\alpha, \chi(k)>=i \int(d x) e^{-i k x}\left(\partial^{2}+\xi M^{2}\right)<\beta\right| \chi^{\star}(x) \mid \alpha>=i(2 \pi)^{4} \delta^{4}\left(p_{\beta}-p_{\alpha}-k\right) \mathcal{M}_{\chi}(\beta ; \alpha, \chi(k))
$$

For a vector boson of polarization $\lambda$

$$
\begin{aligned}
<\beta \mid \alpha, a(k, \lambda)>= & -i \int(d x) e^{-i k x} \varepsilon^{\mu}(k, \lambda) K_{\mu \nu}<\beta\left|A^{\nu^{*}}(x)\right| \alpha> \\
& =i(2 \pi)^{4} \delta^{4}\left(p_{\beta}-p_{\alpha}-k\right) \varepsilon^{\mu} \mathcal{M}_{\mu}(\beta ; \alpha, a[k, \lambda))
\end{aligned}
$$

where

$$
K_{\mu \nu}=g_{\mu \nu}\left(\partial^{2}+M^{2}\right)+\left(\frac{1}{\xi}-1\right) \partial_{\mu} \partial_{\nu}
$$

has the properties

$$
K_{\mu \rho} d^{\rho \nu}(x)=\delta_{\mu}^{\nu} \delta^{4}(x), \quad \partial^{\mu} K_{\mu \nu}=\left(\frac{1}{\xi} \partial^{2}+M^{2}\right) \partial_{\nu}
$$

For longitudinal polarization

$$
\varepsilon^{\mu}\left(k, \lambda_{\ell}\right)=\left(\frac{k}{M}, \hat{k} \frac{\omega}{M}\right)=\frac{k^{\mu}}{M}+\Delta^{\mu}, \quad \Delta^{\mu}=\mathcal{O}\left(\frac{M}{\omega}\right)
$$

So

$$
\mathcal{M}_{\ell}(\cdots)=\varepsilon^{\mu}\left(k, \lambda_{\ell}\right) \mathcal{M}_{\mu}(\cdots)=\frac{k^{\mu}}{M} M_{\mu}(\cdots)\left(1+\mathcal{O} \frac{M^{2}}{\omega^{2}}\right)
$$

Then recalling that $A^{\star}=A$ and using (28) and (23)

$$
\begin{array}{r}
i(2 \pi)^{4} \delta^{4}(\cdots) \frac{k^{\mu}}{M} \mathcal{M}_{\mu}\left(\beta ; \alpha, a\left(k, \lambda_{\ell}\right)\right)=\frac{(-i)^{2}}{M} \int(d x) e^{-i k x}\left(\frac{1}{\xi} \partial^{2}+M^{2}\right)<\beta\left|\partial_{\nu} A^{\nu^{\star}}(x)\right| \alpha> \\
=\int(d x) e^{-i k x}\left(\partial^{2}+\xi M^{2}\right)<\beta\left|\chi^{\star}(x)\right| \alpha>=i(2 \pi)^{4} \delta^{4}(\cdots)(-i) \mathcal{M}(\beta ; \alpha, \chi(k))(31
\end{array}
$$

This provides the result

$$
\frac{k^{\mu}}{M} \mathcal{M}_{\mu}\left(\beta ; \alpha, a\left(k, \lambda_{\ell}\right)=-i \mathcal{M}(\beta ; \alpha, \chi(k))\right.
$$

and the high energy limit for a longitudinaly polarized vector boson

$$
\mathcal{M}\left(\beta ; \alpha, a\left(k, \lambda_{\ell}\right)=-i \mathcal{M}(\beta ; \alpha, \chi(k))\left(1+\mathcal{O} \frac{M^{2}}{\omega^{2}}\right)\right.
$$

Note that the LSZ derivation specifies that in (32), (33) the $\mathrm{k}$ in $a\left(k, \lambda_{\ell}\right)$ and the $\mathrm{k}$ in $\chi(k)$ are the same four- vector. From (24) we see that for arbitrary $\xi$ this can only be satsfied in the limit $M^{2}=0$. The one important exception [⿴囗十 is the Feynman gauge $(\xi=1)$ for which the vector boson and the wouldbe Goldstone boson are on 
the same mass shell, $k^{2}=M^{2}$. We also note that even in Feynman gauge, the left hand side of (32) is an S-Matrix element only in the limit because it is not on the "spin-1 shell". A term proportional to $k_{\mu}$ in $\mathcal{M}_{\mu}$ will contribute a term proportional to $M^{2}$ to $k^{\mu} \mathcal{M}_{\mu}$, but zero to the S-Matrix element $\varepsilon^{\mu} \mathcal{M}_{\mu}$.

For two external vector bosons, the LSZ manipulations are a little more involved, but again follow from (23),(28), without requiring any referennce to Ward identities involving ghost propagators or vertices. Start from

$$
\begin{aligned}
& i(2 \pi)^{4} \delta^{4}(\cdots) \frac{k_{2}^{\nu}}{M} \frac{k_{1}^{\mu}}{M} \mathcal{M}_{\mu \nu}\left(\beta ; \alpha, a\left(k_{2}, \lambda_{\ell}\right), a\left(k_{1}, \lambda_{\ell}\right)\right) \\
= & \frac{k_{2}^{\nu}}{M} \frac{k_{1}^{\mu}}{M}(-i)^{2} \iint\left(d x^{\prime}\right)(d x) e^{-i\left(k_{2} x^{\prime}+k_{1} x\right)} K_{\nu \tau}^{\prime} K_{\mu \sigma}<\beta\left|T\left(A^{\tau}\left(x^{\prime}\right) A^{\sigma}(x)\right)\right| \alpha>
\end{aligned}
$$

The k's are converted to derivatives on the A's by partial integration from the exponentials and use of (28). (We neglect possible contact terms which don't contribute to the S-matrix). Each $\partial A$ is replaced by $F-X$ where $X \equiv \xi M \chi^{\star}(21)$. This generates four terms. The FF term is proportional to the matrix element

$$
<\beta \mid T\left(F\left(\left(x^{\prime}\right) F(x)\right)|\alpha>=-\xi<\beta| T\left(\left\{Q, \bar{c}\left(x^{\prime}\right)\right\} F(x)\right) \mid \alpha>\right.
$$

which vanishes because the BRST charge Q commutes with F and anihilates the physical states $<\beta \mid$ and $\mid \alpha>$. An FX term is of the form

$$
\begin{aligned}
& \frac{1}{M} \int\left(d x^{\prime}\right) e^{-i k_{2} x^{\prime}}\left(\frac{1}{\xi} \partial^{\prime 2}+M^{2}\right) \\
& \quad \times \int(d x) e^{-i k_{1} x}\left(\partial^{2}+\xi M^{2}\right)<\beta\left|T\left(F\left(x^{\prime}\right) \chi^{\star}(x)\right)\right| \alpha>
\end{aligned}
$$

The integral on the second line is just the reduction formula for $\chi^{\star}$. Run back it is equal to

$$
-i<\beta\left|F\left(x^{\prime}\right)\right| \alpha, \chi\left(k_{1}\right)>=0
$$

The XX term is

$$
\begin{array}{r}
-i^{2} \int\left(d x^{\prime}\right) \int(d x) e^{-i\left(k_{2} x^{\prime}+k_{1} x\right)}\left(\partial^{\prime 2}+\xi M^{2}\right)\left(\partial^{2}+\xi M^{2}\right)<\beta\left|T\left(\chi^{\star}\left(x^{\prime}\right) \chi^{\star}(x)\right)\right| \alpha> \\
=-i(2 \pi)^{4} \delta^{4}(\cdots) \mathcal{M}\left(\beta ; \alpha, \chi\left(k_{2}\right), \chi\left(k_{1}\right)>\right.
\end{array}
$$

Matching this to the start of (34) gives the result

$$
\frac{k_{2}^{\nu}}{M} \frac{k_{1}^{\mu}}{M} \mathcal{M}_{\mu \nu}\left(\beta ; \alpha, a\left(k_{2}, \lambda_{\ell}\right), a\left(k_{1}, \lambda_{\ell}\right)\right)=-\mathcal{M}\left(\beta ; \alpha, \chi\left(k_{2}\right) \chi\left(k_{1}\right)\right)
$$

and the high energy limit for two longitudinaly polarized vector bosons

$$
\mathcal{M}\left(\beta ; \alpha, a\left(k_{2}, \lambda_{\ell}\right), a\left(k_{1}, \lambda_{\ell}\right)\right)=-\mathcal{M}\left(\beta ; \alpha, \chi\left(k_{2}\right), \chi\left(k_{1}\right)\right)\left(1+\mathcal{O} \frac{M^{2}}{\omega^{2}}\right)
$$

The remarks made after (32), (33) apply as well to (36), (37). At first sight, this appears to say that the Equivalence Theorem holds without modification order by order in the perturbative loop expansion. But we still have to relate the renormalized 
$\tau^{\star}-$ functions $<0\left|T\left(\phi^{\star} \ldots\right)\right| 0>$ appearing in the LSZ reduction formulas to the renormalized $\tau-$ functions $<0 \mid T(\phi \cdots \mid 0>$ calculated according to section two, to maintain the BRST structure defined in the unrenormalized theory. This is ( $\phi$ is any field in the theory)

$$
\tau^{\star}(\cdots)=<0\left|T\left(\phi^{\star} \cdots\right)\right| 0>=\sqrt{\frac{Z_{\phi}}{Z_{\phi}^{\star}}} \cdots<0|T(\phi \cdots)| 0>
$$

So when the perturbative calculation of the S-matrix elements (the $\mathcal{M}$ 's) is given in terms of $\tau-$ functions, a factor $\sqrt{\frac{Z_{\phi}}{Z_{\phi}^{\star}}}$ will appear for each $\phi$ which is not starnormalized, $\left(Z_{\phi} \neq Z_{\phi}^{\star}\right)$. In particular, in the renormalization scheme of section two, a single $\mathrm{Z}$ is used to renormalize both $h_{0}$ and $\chi_{0}$. In the case of Spontaneous Symmetry Breaking, $Z_{h}^{\star} \neq Z_{\chi}^{\star}$ so it is not possible for both $\hat{h}$ and $\chi$ to be star-normalized. There is yet another factor which has to be taken into acount. For S-matrix elements computed from $\tau^{\star}-$ functions, there are no loop corrections to external lines. LSZ amputation by the inverse free propagator removes the complete on-shell external propagator because it has unit residue at its pole. When S-matrix elements are computed from $\tau$-functions, there are loop corrections to external lines which survive the LSZ amputation. These are of the form

$$
\begin{gathered}
-i\left(p^{2}-m^{2}\right)\left\{\frac{i}{p^{2}-m^{2}}+\frac{i}{p^{2}-m^{2}}\left(-i \Sigma^{F D}\left(p^{2}\right)+i \delta Z\left(p^{2}-m^{2}\right)-\delta m^{2}\right) \frac{i}{p^{2}-m^{2}}+\cdots\right\} \underline{\tilde{\mathcal{\tau}}} \\
=\left\{1+\left(\frac{\partial \Sigma^{F D}}{\partial p^{2}}-\delta Z\right)\right\} \tilde{\tilde{\tau}}=\left\{1+\delta Z^{\star}-\delta Z+\cdots\right\} \tilde{\tilde{\tau}}=\frac{Z^{\star}}{Z} \tilde{\tilde{\tau}}
\end{gathered}
$$

This factor just reverses that of (38) So the final result is

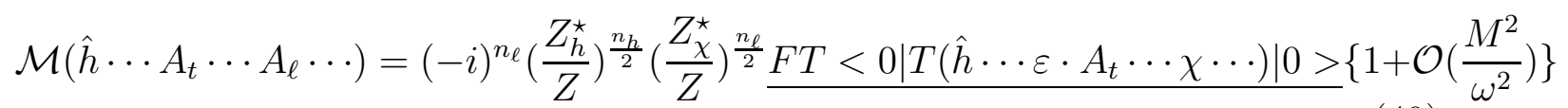

The FT is fourier transform with four-momentum delta function factored off; and the underline means complete external line propagators removed.

$$
\tilde{\tau}(\cdots)_{n}=(i D)^{n} \tilde{\tau}(\cdots)_{n}
$$

The result (40) is simple, intuitive, and quite general. (If the vector boson twopoint function is not normalized to unit residue, (40) also includes $\sqrt{\frac{Z_{A}^{\star}}{Z_{A}}}$ factors). For $k^{\mu} k_{\mu}=M^{2}$, the left hand side is an S-matrix element and must be independent of $\xi$. In the high energy limit, in the leading order, there is no contribution from the $\mathcal{O}\left(M^{2} / \omega^{2}\right)$ terms on right hand side and in this leading order, the product of ratios of $\mathrm{Z}$ factors and the amputated $\tau$-function (which product is just the on-shell $\tau^{\star}$ function) must be independent of $\xi$. In section five we carry out an explicit one-loop perturbative calculation to check that the correction factors in (40) are indeed correct.

There is still some freedom of choice of renormalization conditions to complete the specification of the renormalization (7). To conclude this section, we recall the choices we have made in order to arrive at the results (36), (40). (1) The gauge fixing function F 
is chosen to cancel tree level $\chi-\partial A$ mixing. ( $\kappa=1+$ one-loop). (2)Renormalization (17) is constrained to maintain BRST symmetry in same form as unrenormalized. (3)Renormalized masses are the physical masses (modulo unstable particles. See discussion below). (4)The vector boson $\mathrm{A}$ is renormalized to satisfy the unit residue condition $\left(Z_{A}=Z_{A}^{\star}\right)$. (5)A specific choice of the second gauge parameter $\kappa$ is made to get (36) to come out with no extra factors. Since the end results (37), (40) are Smatrix elements, presumably other renormalization schemes are admissable, but the one outlined here seems the simplest and most physical and intuitive. (See also the example explicit calculation in section five, and also of course, alternative treatments from [1] and references cited therein).

\section{Decay of an Unstable Particle and the Equiva- lence Theorem}

Let a, $\chi$ be generic vector bosons and wouldbe Goldstone bosons, with masses squared $M^{2}$ and $\xi M^{2}$ and $m=m_{h}$ the Higgs boson mass. If $m_{h}>2 m_{a}$, the Higgs boson is unstable and the standard calculation of the decay rate for $h \rightarrow a_{\ell} a_{\ell}$ is given by

$$
\Gamma=\frac{1}{2 m}\left|\mathcal{M}\left(h ; a\left(k_{1}, \lambda_{\ell}\right), a\left(k_{2}, \lambda_{\ell}\right)\right)\right|^{2} \frac{1}{2} \int d \Phi
$$

If the Equivalence Theorem applies, (In this case, the high energy limit is just the mass of Higgs boson much greater than the masses of the gauge bosons or wouldbe Goldstone bosons ) one may substitute for $\mathcal{M}$ in (42) the invariant matrix- element for decay into two wouldbe Goldstone bosons .

Since the $\mathrm{h}$ particle passes from stable to unstable by an arbitrarily small change in the continuous parameter $m^{2} / M^{2}=2 \lambda / g^{2}$ from $<4$ to $>4$, continuity suggests that the form of $\mathcal{M}$ should be unchanged as the pole of the $\mathrm{h}$ propagator moves continuously into the second sheet. [3]

A derivation based on unitarity of the resonance contribution to elastic scattering goes as follows. With $M^{2}, \xi M^{2}<m^{2}$, the vector boson and the wouldbe Goldstone boson are stable. So we can apply the Equivalence Theorem to the elastic scattering processes $a_{\ell}+a_{\ell} \rightarrow a_{\ell}+a_{\ell}, \quad a_{\ell}+\chi \rightarrow a_{\ell}+\chi, \chi+\chi \rightarrow \chi+\chi$ in the limit $M^{2} \ll \omega_{i}^{2}$.

Unitarity for the invariant matrix element $\mathcal{M}$ for forward elastic scattering of two identical spinless particles, $\chi+\chi \rightarrow \chi+\chi$, is

$$
2 \Im \mathcal{M}_{\alpha \alpha}=\frac{1}{2} \int d \Phi_{2}\left|\mathcal{M}_{\alpha \alpha}\right|^{2} \quad(\alpha=\chi \chi)
$$

For a narrow resonance

$$
\begin{array}{r}
\mathcal{M}=\mathcal{M}_{\text {res }}+\mathcal{M}_{b g d} \\
\mathcal{M}_{r e s}=-\frac{R^{2}}{s-m_{p}^{2}+i m_{p} \Gamma}
\end{array}
$$

Consider summed renormalized perturbation theory.(skeleton expansion) 

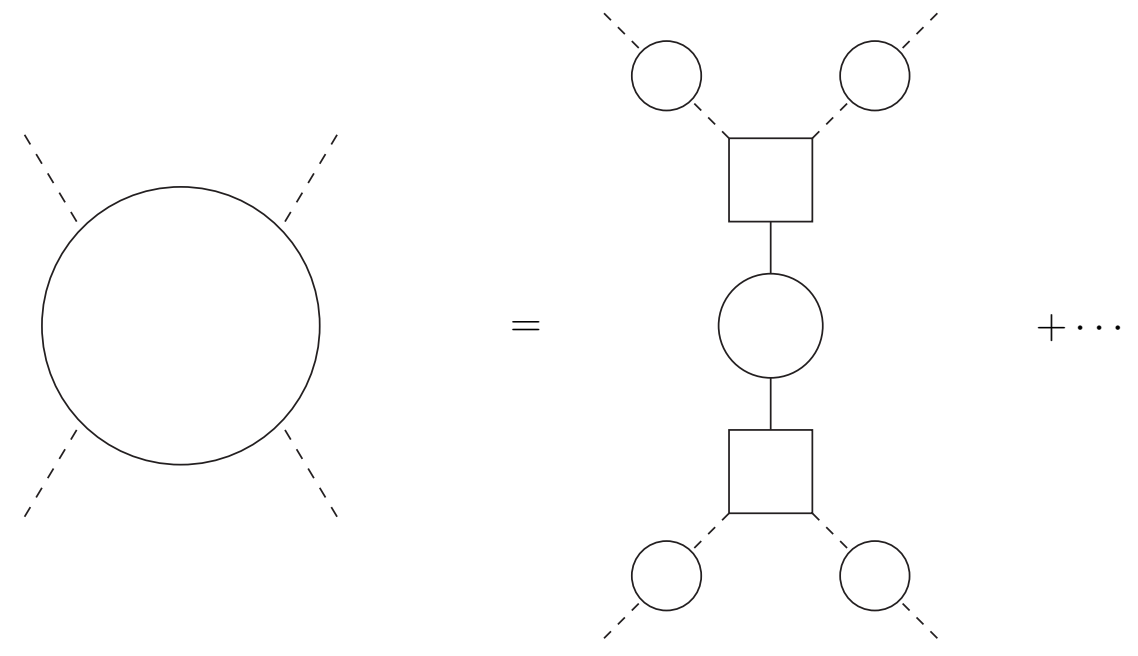

Fig.1. Resonance at complex pole of summed propagator of unstable particle

$$
\tau=\left(i D_{\chi}\right)^{4}(i V)^{2} i D_{h}=\left(\frac{Z_{\chi}^{\star}}{Z_{\chi}}\right)^{2} \tau^{\star}=\left(\frac{Z_{\chi}^{\star}}{Z_{\chi}}\right)^{2}\left(i D_{\chi}^{\star}\right)^{4}\left(i V^{\star}\right)^{2} i D_{h}^{\star}
$$

$\mathrm{V}$ is the completely amputated 1PI $h \chi \chi$ vertex function (diagrams in the third columns of figures three and four).

$$
V=\frac{Z_{\chi}}{Z_{\chi}^{\star}}\left(\frac{Z_{h}}{Z_{h}^{\star}}\right)^{\frac{1}{2}} V^{\star}
$$

The summed h-propagator is

$$
D_{h}=\frac{Z_{h}^{\star} / Z_{h}}{k^{2}-m_{r}^{2}-\Re \Sigma_{h}^{\star}{ }^{\star}\left(k^{2}\right)-i \Im \Sigma_{h}^{\star}\left(k^{2}\right)}
$$

The double bar indicates two subtractions (in addition to multiplicative renormalization) for $\Re \Sigma$. The first subtraction secures $\Re \Sigma\left(m_{r}^{2}\right)=0$. The second subtraction makes the derivative of $\Re \Sigma\left(k^{2}\right)$ vanish at $k^{2}=m_{r}^{2}$.

We observe that the renormalized mass $m_{r}^{2}$ defined by these subtractions is not the same as the mass $m_{p}^{2}$ which is a parameter of the exact pole position in (44). The difference is

$$
m_{p}^{2}-m_{r}^{2}=\mathcal{R} e\left(\Re \overline{\bar{\Sigma}}\left(s_{p}\right)+i \Im \Sigma\left(s_{p}\right)\right)
$$


This difference is second order in the expansion parameter of the theory

$$
g^{2} \frac{m^{2}}{M^{2}}, \quad\left(\frac{\Gamma}{m} \sim g^{2} \frac{m^{2}}{M^{2}}\right)
$$

So the difference between these two renormalized masses can be ignored in a first order perturbative calculation. But the difference between the complex pole position and the real mass squared is a first order imaginary quantity $(-i m \Gamma)$ which will be essential for the verification of the Equivalence Theorem .

Applying LSZ, the resonance contribution to the invariant matrix element for $\chi+\chi \rightarrow \chi+\chi$ is

$$
\begin{aligned}
i \mathcal{M}_{\text {res }}= & i V^{\star} i D_{h}^{\star} i V^{\star}=\quad\left(\frac{Z_{\chi}^{\star}}{Z_{\chi}}\right)^{2} i V i D_{h} i V \\
= & i \frac{-\left(\frac{Z_{\chi}^{\star}}{Z_{\chi}} \sqrt{\frac{Z_{h}^{\star}}{Z_{h}}} V\right)^{2}}{s-m_{r}^{2}-i \Im \Sigma_{h}^{\star}\left(m_{r}^{2}\right)} \equiv i \frac{-(f V)^{2}}{s-m_{r}^{2}+i m_{r} \Gamma}
\end{aligned}
$$

Substitute this into the unitarity equation (43)

$$
\begin{array}{r}
2 \Im \mathcal{M}_{\alpha \alpha}=2 \frac{(f V)^{2} m_{r} \Gamma}{\left(s-m_{r}^{2}\right)^{2}+m_{r}^{2} \Gamma^{2}} \\
=\frac{1}{2} \int d \Phi_{2}\left|\mathcal{M}_{\alpha \alpha}\right|^{2}=\frac{1}{2} \int d \Phi_{2} \frac{(f V)^{4}}{\left(s-m_{r}^{2}\right)^{2}+m_{r}^{2} \Gamma^{2}}
\end{array}
$$

which implies

$$
\Gamma=\frac{1}{2 m_{h}}|f V|^{2} \frac{1}{2} \int d \Phi_{2}
$$

Repeating the unitarity calculation for the other two initial/final states, $\chi a_{\ell}, a_{\ell} a_{\ell}$ gives two more versions of (50), each with $\mathrm{f}$ being the product of ratios of renormalization constants required to convert amputated $\tau-$ functions to S-Matrix elements . Through one-loop the perturbabtive calculation of the 1PI vertex function encounters no multiple poles (no Dyson resummation required). And for the calculation of the Higgs decay rate, the real part of the variable $k^{2}$ is fixed at $m_{r}^{2}$ i.e. at the resonance peak; so the calculation does not depend on choice of off-peak representation (e.g. partial wave threshold factors) of the resonant scattering amplitude.

To check the applicability of the Equivalence Theorem to the calculation of the decay rate of an unstable particle, the tree plus one-loop perturbative calculations of the 1PI vertex functions $V_{h, a_{\ell}, a_{\ell}}, V_{h, a_{\ell}, \chi}, V_{h, \chi, \chi}$ are given in the next sections.

\section{Perturbative Calculation in the Abelian Higgs model}

We complete the specification of the renormalization by enumerating the parameters and renormalization conditions and renormalization constants .

The renormalized parameters (17) on which physical quantities can depend are

$$
\mu^{2}, \lambda, \quad g
$$


There are two gauge parameters

$$
\xi, \kappa
$$

The gauge parameter $\kappa$ has been fixed by considerations given above, equation (19). The gauge parameter $\xi$ is left free. The corresponding renormalization constants are

$$
Z_{\mu^{2}}, Z_{\lambda}, Z_{g}, Z_{\xi}, Z_{\kappa}
$$

The field strength renormalization constants are

$$
Z_{A}=Z_{A}^{\star}, Z
$$

$Z_{\kappa}$ is fixed for consistent renormalization of the gauge fixing function $\mathrm{F}$ (12), $Z_{\xi}, Z_{g}$ are related to $Z_{A}$ by Ward identites (9). There is also $\mathrm{V}$, which has to be determined as a function of the renormalized parameters of the theory (including $\xi$ ). We introduce $\zeta_{v}$ by the relation

$$
\begin{gathered}
V=\zeta_{v} v=v+\left(\zeta_{v}-1\right) v \\
v^{2}=\frac{\left(-\mu^{2}\right)}{\lambda}, \quad \zeta_{v}=1+\zeta_{v}^{\text {one-loop }}+\cdots
\end{gathered}
$$

We trade $\mu^{2}, \lambda, g$ for $m, M, g$

$$
m^{2}=2\left(-\mu^{2}\right), \quad M^{2}=g^{2} \frac{\left(-\mu^{2}\right)}{\lambda}, \quad\left(\lambda=\frac{g^{2}}{2} \frac{m^{2}}{M^{2}}\right)
$$

So we require five conditions to fix the four renormalization constants $Z_{\mu^{2}}, Z_{\lambda}, Z_{A}, Z$ and $\zeta_{v}$ (the vev).

(1)All tadpoles are canceled order by order

$$
<\hat{h}>=i T=0
$$

(2)The induced $\chi-\partial A$ mixing vanishes at $k^{2}=\xi M^{2}=M_{\chi}^{2}$

$$
X^{\mu}=k^{\mu} X\left(k^{2}\right), \quad X\left(\xi M^{2}\right)=0
$$

(3),(4)The gauge invariant part of the complete renormalized vector boson propagator has pole at the physical mass with unit residue.

$$
\Pi_{T}\left(M^{2}\right)=0, \quad \frac{d \Pi_{T}\left(M^{2}\right)}{d k^{2}}=0
$$

(5) The stable h propagtor has pole at the physical mass.

$$
\Sigma_{h}\left(m^{2}\right)=0, \quad \text { for unstable h } \Re \Sigma_{h}\left(m_{r}^{2}\right)=0
$$

Note that neither condition, $\frac{d \Sigma_{h}\left(m^{2}\right)}{d k^{2}}=0$ or $\frac{d \Sigma_{\chi}\left(\xi M^{2}\right)}{d k^{2}}=0$ is included. Thus Z is not required to be equal to either $Z_{h}^{\star}$ or $Z_{\chi}^{\star}$.

In these equations, each of the functions $T, X, \Pi_{T}, \Sigma_{h}$ is a renormalized $1 P I$ function. Each has two components

$$
f=f^{F D}+f^{c t}
$$


The first is the sum of (one-loop) Feynman diagrams with renormalized masses in the free propagators and renormalized coupling constants at the elementary vertices. The Feynman integrals are evaluated with dimensional regularization. The second is the corresponding tree diagram multiplied by $\left(Z_{i}-1\right)$ and $\left(\zeta_{v}-1\right)$ factors.

The renormalization constants and $\zeta_{v}$ which are fixed by these conditions are given in appendix $\mathrm{A}$.

(5')With no adjustable parameter

$$
\Sigma_{\chi}\left(\xi M^{2}\right)_{M^{2} \rightarrow 0} \rightarrow 0
$$

The limit $M^{2} \rightarrow 0, g^{2} \rightarrow 0, v^{2}=\frac{-\mu^{2}}{\lambda}$ fixed is the "gaugeless" limit in which the broken symmetry is a global symmetry and $\chi$ is a genuine Goldstone boson. Since we have been careful to renormalize consistent with the unbroken symmetry, the Goldstone boson remains massless after renormalization. [5] We see that in the context of the Abelian Higgs model, the gaugeless limit could as well be called the Goldstone limit. Furthermore, the Equivalence Theorem is supposed to hold in the limit of energies much greater than the gauge boson masses. For the decay of the Higgs, the energy is the Higgs mass and the condition $\frac{M^{2}}{m^{2}} \ll 1$ is just this limit. The result $\delta \zeta_{v}=0$ is special to this limit, in which the Goldstone theorem provides for the same counter term to eliminate both the tadpoles and the Goldstone boson mass. When the gauge interactions are included, $\delta \zeta_{v}$ is both gauge dependent and ultraviolet divergent.

We turn now to the explicit calculation of the decay probabilites (42) for $h \rightarrow$ $a, a, \quad h \rightarrow a, \chi, \quad h \rightarrow \chi, \chi$. For these processes, $\omega$ is $m / 2$. To check the Equivalence Theorem, we compute to leading order in powers of $M^{2} / m^{2}$. In this order, the longitudinal polarization vectors are replaced by $k^{\mu} / \mathrm{M}$. At tree-level, the invariant matrix elements (the $\mathcal{M}^{\prime} s$ ) are equal to the tree-level $\tilde{\tau}^{\prime} s$ which are just the elementary vertices contracted with $k^{\mu} / \mathrm{M}$ for each external vector boson.(Fig.2.) (The ratios of $\mathrm{Z}$ factors differ from one only at one-loop order).
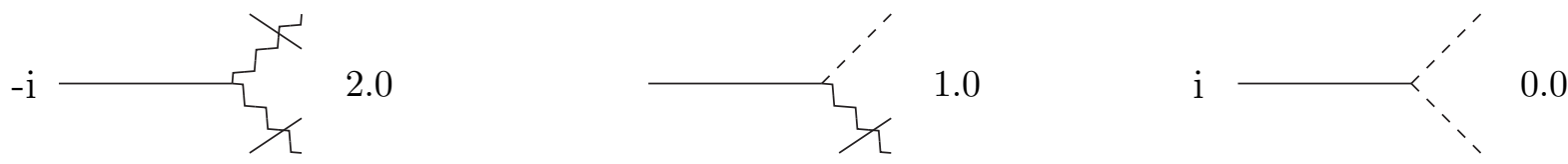

Fig.2.Tree diagrams for $h \rightarrow a_{\ell} a_{\ell}, \chi a_{\ell}, \chi \chi$.

The slashes indicate contraction with $k^{\mu} / M$.

For decay into two longitudinal vector bosons:

$$
-i \frac{k_{2}^{\nu}}{M} \frac{k_{1}^{\mu}}{M} \tilde{\tau}_{\mu \nu}=2 g M \frac{k_{1} \cdot k_{2}}{M^{2}}
$$

But in the limit $M^{2} \rightarrow 0$,

$$
2 k_{1} \cdot k_{2}=K^{2}
$$

the square of the four-momentum of Higgs boson, which is to be evaluated at its complex on-shell value,

$$
K^{2}=m^{2}-i m \Gamma
$$


Substituting the zeroth order $m^{2}$ into (42) gives the first approximation to $\Gamma$

$$
\Gamma=\frac{g^{2}}{32 \pi} \frac{m^{3}}{M^{2}}
$$

Substitute all this into (58)to obtain

$$
-i \frac{k_{2}^{\nu}}{M} \frac{k_{1}^{\mu}}{M} \tilde{\tau}_{\mu \nu}=g \frac{m^{2}}{M}-i \pi \frac{g^{3}}{16 \pi^{2}} \frac{m^{4}}{M^{3}} \frac{1}{2} .
$$

For decay into one vector boson and one wouldbe Goldstone Boson

$$
\frac{k_{2}^{\nu}}{M} \tilde{\mathcal{T}}_{\nu}=\frac{k_{2}^{\mu}}{M} g\left(2 k_{1}-k_{2}\right)_{\mu}=2 g \frac{k_{1}^{\mu} \cdot k_{2}^{\mu}}{M}
$$

which is identical to (58). For decay into two wouldbe Goldstone bosons, the treelevel invariant matrix-element is simply

$$
i \tilde{\tilde{\tau}}=g \frac{m^{2}}{M} .
$$

There is no explicit dependence on the Higgs boson four-momentum; hence no imaginary shift is generated.

For two transversely polarized vector bosons the tree amplitude is just $g M$, down by $M^{2} / m^{2}$. Note that in the "gaugeless" limit, $g, M \rightarrow 0$ while $m^{2} \frac{g}{M}$ remains finite. At one-loop order, the dominant terms are $g^{3} m^{4} / M^{3}$, finite in the limit. Subdominant terms are of order $g^{3} m^{2} / M$ and vanish in the limit. In this limit - which is equvialently the heavy Higgs limit - the trilinear and quartic scalar couplings are dominent over the gauge couplings. All one-loop diagrams with internal gauge vector boson lines are in the subdominent class. This leads to substantial simplification of the calculation, and, along with the observation that the $\chi$ mass squared $\xi M^{2}$ also disapears in the limit, shows that there is no source of $\xi$ dependence in the calculation in this limit.

In figures 3 and 4 are shown the one-loop Feynman diagrams and counterterm diagrams which contribute in this limit to the $\tilde{\tau}$ functions whose tree-level values are given in (60, 62). The corresponding calculated values are given in Table 1. 


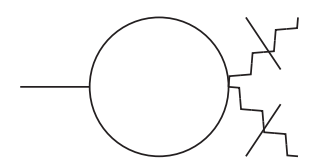

2.a

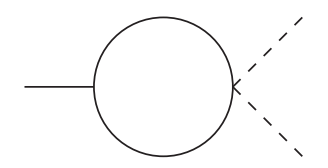

0.a
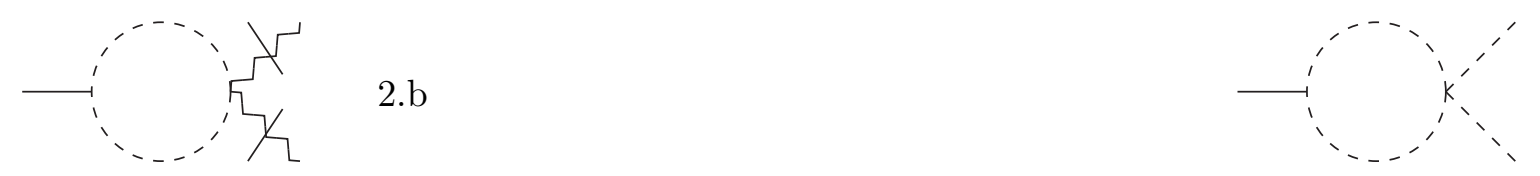

0.b

[2]

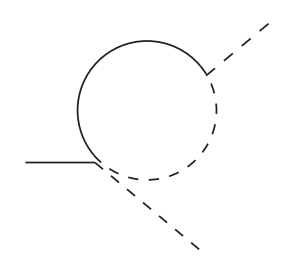

0.c

2.d
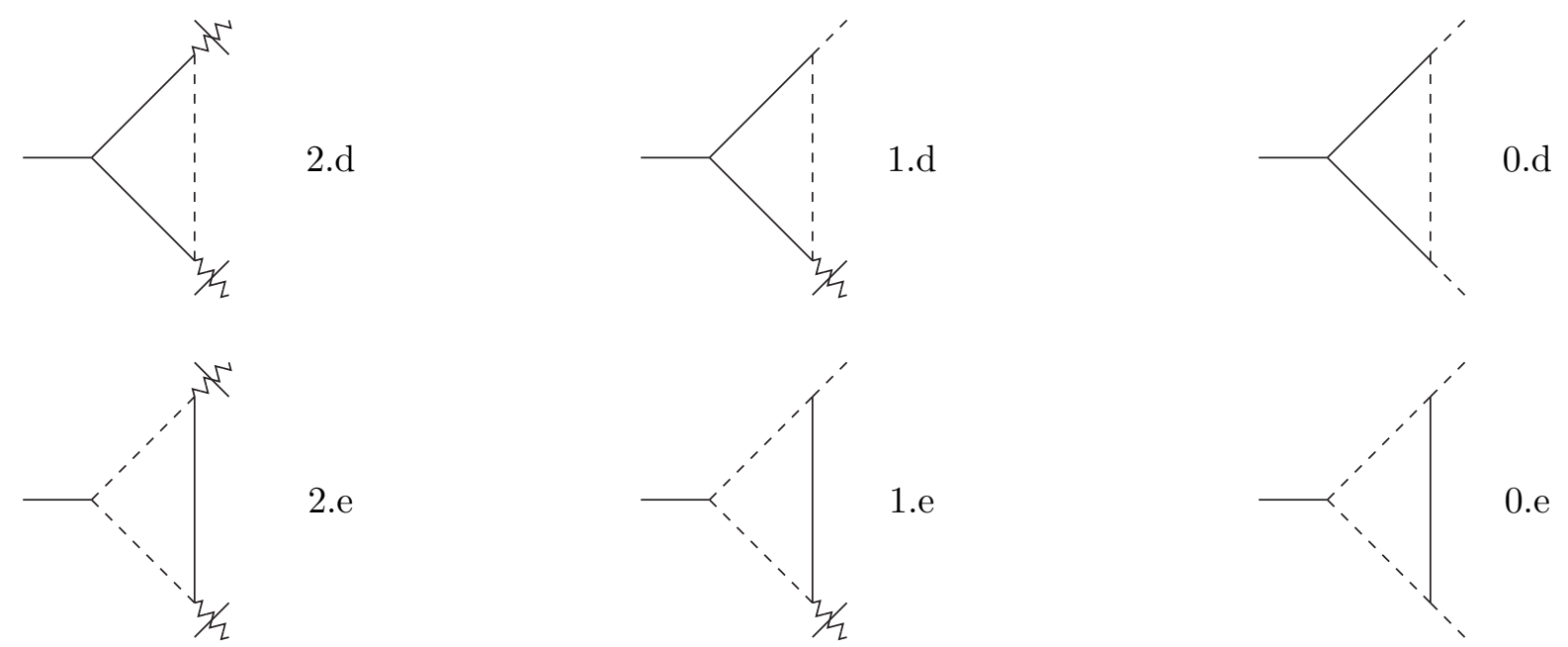

Fig.3. One-loop diagrams which contribute to $h \rightarrow a_{\ell} a_{\ell}, \chi a_{\ell}, \chi \chi$ in the limit $g \rightarrow 0, M \rightarrow 0, g / M=1 / v$. In this limit there are no contributions 2.c,1.a,1.b,1.c

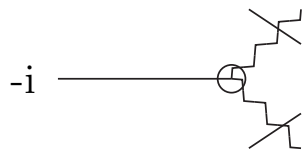

$\left(Z_{4 g} \zeta_{v}-1\right)$

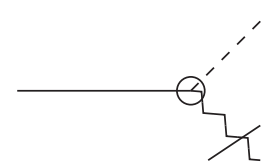

$\left(Z_{3 g}-1\right)$

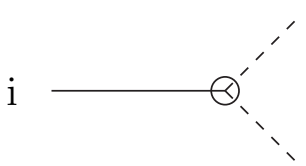

$\left(Z_{4 \lambda} \zeta_{v}-1\right)$

Fig.4. One-loop counterterm diagrams. Each factor displayed is multiplied by common factor $g \frac{m^{2}}{M}$ 


$$
\begin{array}{|c|c|c|c|} 
& -i \frac{k_{2}^{\nu}}{M} \frac{k_{1}^{\mu}}{M} \tilde{\tau}_{\mu \nu} & \frac{k_{2}^{\nu}}{M} \tilde{\tau}_{\nu} & i \tilde{\tau} \\
\hline \mathrm{a} & -\frac{3}{2} \Delta_{\epsilon}-3+\frac{\sqrt{3}}{2} \pi & & -\frac{3}{2} \Delta_{\epsilon}-3+\frac{\sqrt{3}}{2} \pi \\
\mathrm{b} & -\frac{1}{2} \Delta_{\epsilon}-1+i \pi\left(-\frac{1}{2}\right) & & -\frac{3}{2} \Delta_{\epsilon}-3+i \pi\left(-\frac{3}{2}\right) \\
\mathrm{c} & & & 2\left[-\Delta_{\epsilon}-1\right] \\
\mathrm{d} & \frac{3}{2} \Delta_{\epsilon}+6-\frac{3 \sqrt{3}}{2} \pi+\frac{\pi^{2}}{3} & 3-\sqrt{3} \pi+\frac{1}{3} \pi^{2} & \frac{\pi^{2}}{3} \\
\mathrm{e} & \frac{1}{2} \Delta_{\epsilon}+\frac{\pi^{2}}{12}+i \pi\left(-\frac{1}{2}+\ln 2\right) & -1+\frac{\pi^{2}}{12}+i \pi(-1+\ln 2) & \frac{\pi^{2}}{12}+i \pi(\ln 2) \\
\mathrm{ct} & -\frac{1}{2} & -\frac{1}{2} & 5 \Delta_{\epsilon}+\frac{19}{2}-\frac{3 \sqrt{3}}{2} \pi
\end{array}
$$

Table 1: The one-loop vertex contributions in the Abelian Higgs model. Each entry is multiplied by common factor $\frac{g^{3}}{16 \pi^{2}} \frac{m^{4}}{M^{3}}$

The result of adding all the contributions to each of these is

$$
\begin{gathered}
-i \frac{k_{2}^{\nu}}{M} \frac{k_{1}^{\mu}}{M} \tilde{\mathcal{I}}_{\mu \nu}=g \frac{m^{2}}{M}\left\{1+\frac{g^{2}}{16 \pi^{2}} \frac{m^{2}}{M^{2}}\left[\frac{3}{2}-\sqrt{3} \pi+\frac{5}{12} \pi^{2}+i \pi(\ln 2-1)\right]+\cdots\right\} \\
\frac{k_{2}^{\nu}}{M} \tilde{\mathcal{\tau}}_{\nu}==g \frac{m^{2}}{M}\left\{1+\frac{g^{2}}{16 \pi^{2}} \frac{m^{2}}{M^{2}}\left[\frac{3}{2}-\sqrt{3} \pi+\frac{5}{12} \pi^{2}+i \pi(\ln 2-1)+\cdots\right]\right\} \\
i \underline{\tilde{\tau}}=g \frac{m^{2}}{M}\left\{1+\frac{g^{2}}{16 \pi^{2}} \frac{m^{2}}{M^{2}}\left[\frac{3}{2}-\sqrt{3} \pi+\frac{5}{12} \pi^{2}+i \pi\left(\ln 2-\frac{3}{2}\right)+\cdots\right]\right\}
\end{gathered}
$$

which differ in the imaginary parts. To get the $\mathcal{M}$ 's to put into (42), we have to multiply by the factors of ratios of square roots of Z's required to convert amputated $\tau-$ functions, renormalized as described above, to S-Matrix elements . Using the ratios given in appendix $\mathrm{A}$, these factors are

$$
\begin{gathered}
\sqrt{\frac{Z_{h}^{\star}}{Z}}=1+\frac{g^{2}}{16 \pi^{2}} \frac{m^{2}}{M^{2}}\left[\frac{11}{4}-\frac{\sqrt{3}}{2} \pi\right] \\
\sqrt{\frac{Z_{h}^{\star}}{Z} \sqrt{\frac{Z_{\chi}^{\star}}{Z}}}=1+\frac{g^{2}}{16 \pi^{2}} \frac{m^{2}}{M^{2}}\left[\frac{11}{4}-\frac{\sqrt{3}}{2} \pi\right] \\
\sqrt{\frac{Z_{h}^{\star}}{Z}} \frac{Z_{\chi}^{\star}}{Z}=1+\frac{g^{2}}{16 \pi^{2}} \frac{m^{2}}{M^{2}}\left[\frac{11}{4}-\frac{\sqrt{3}}{2} \pi\right]
\end{gathered}
$$

which are all the same because of the special circumstance that $Z=Z_{\chi}^{\star}$ And we have to include the imaginary parts generated from the tree-level amplitudes by evaluating at the complex four-momentum squared (60) When all the factors are combined, the three invariant matrix elements are now the same.

$$
\begin{gathered}
\frac{k_{2}^{\nu}}{M} \frac{k_{1}^{\mu}}{M} \mathcal{M}_{\mu \nu}=i \frac{k_{2}^{\nu}}{M} \mathcal{M}_{\nu}=(-1) \mathcal{M} \\
=g \frac{m^{2}}{M}\left\{1+\frac{g^{2}}{16 \pi^{2}} \frac{m^{2}}{M^{2}}\left[\frac{17}{4}-\frac{3 \sqrt{3}}{2} \pi+\frac{5}{12} \pi^{2}+i \pi\left(\ln 2-\frac{3}{2}\right)+\cdots\right]\right\}\left(1+\mathcal{O} \frac{M^{2}}{m^{2}}\right)
\end{gathered}
$$




\section{The calculation in the Standard Model}

The BRST structure of the Nonabelian gauge sector of the Standard Model is somewhat more complicated than that of the Abelian Higgs model presented in section two. But the key result (23), used in sections three and four, is maintained; so we go directly to the perturbative calculation

We calculate the decay rate for $h \rightarrow W_{+} W_{-}$and $h \rightarrow \phi_{+} \phi_{-}$through one-loop order in the Standard Model, in the heavy Higgs limit, $m^{2} \gg M^{2}\left(\lambda \gg g^{2}\right)$. We also take the Weinberg angle to be zero. The leading terms in this limit can be calculated starting from a truncated Standard Model Lagrangian.

$$
\begin{gathered}
\mathcal{L}=\frac{1}{2}(\partial \hat{h})^{2}+\left(\partial \phi^{\dagger}\right) \partial \phi+\frac{1}{2}\left(\partial \phi_{0}\right)^{2}-\frac{1}{2} m^{2} \hat{h}^{2} \\
-\lambda v \hat{h}\left(\hat{h}^{2}+2 \phi^{\dagger} \phi+\phi_{0}^{2}\right)-\frac{\lambda}{4}\left(\hat{h}^{2}+2 \phi^{\dagger} \phi+\phi_{0}^{2}\right)^{2} \\
\left.+g M \hat{h} W^{\dagger} W-i \frac{g}{2}\left(\phi^{\dagger} \stackrel{\leftrightarrow}{\partial} \hat{h}\right) W-(\phi \stackrel{\leftrightarrow}{\partial} \hat{h}) W^{\dagger}\right)-\frac{g}{2}\left(\left(\phi \stackrel{\leftrightarrow}{\partial} \phi_{0}\right) W^{\dagger}+\left(\phi^{\dagger} \stackrel{\leftrightarrow}{\partial} \phi_{0}\right) W\right) \\
+\mathcal{L}_{c t} \\
m^{2}=2 \lambda v^{2}, \quad M^{2}=\frac{1}{4} g^{2} v^{2}, \quad \lambda=\frac{g^{2}}{8} \frac{m^{2}}{M^{2}}
\end{gathered}
$$

For the calculation of $h \rightarrow \phi_{+} \phi_{-}$, the purely scalar Lagrangian is sufficient. The calculation of $h \rightarrow W_{+} W_{-}$requires also the couplings to the "external"W field with fixed coupling $g$.

The tree-level for decay into longitudinal $W_{+} W_{-}$is

$$
\frac{k_{2}^{\nu}}{M} \frac{k_{1}^{\mu}}{M} \mathcal{M}_{\mu \nu}=\frac{g}{M} k_{1} \cdot k_{2}=\frac{g}{2 M}\left(m^{2}-i m \Gamma\right)
$$

The width in (66) is the total width, including decay into $Z_{0} Z_{0}$. This is

$$
\Gamma=\frac{g^{2}}{64 \pi} \frac{m^{3}}{M^{2}} \frac{3}{2}
$$

Substiituting this into (66) gives

$$
\frac{k_{2}^{\nu}}{M} \frac{k_{1}^{\mu}}{M} \mathcal{M}_{\mu \nu}=\frac{g}{2} \frac{m^{2}}{M}\left(1-i \pi \frac{g^{2}}{16 \pi^{2}} \frac{m^{2}}{M^{2}} \frac{3}{8}\right)
$$

For decay into $\phi_{+} \phi_{-}$

$$
-\mathcal{M}=\frac{g}{2} \frac{m^{2}}{M}
$$

Again, no imaginary shift is generated because there is no explicit dependence on $K^{2}$,

Renormalization of the truncated Lagrangian is substantially simpler than the renormalization of the complete Electroweak Standard Model [6]. We use the renormalization scheme and renormalization constants from [5]. The Feynman diagrams to be calculated are just those of the first and third columnes of figures 2,3,4, with one addition to the first column of fig.3. Because the Standard Model has both charged 


$$
\begin{array}{|c|c|c|} 
& -i \frac{k_{2}^{\nu}}{M} \frac{k_{1}^{\mu}}{M} \tilde{\tau}_{\mu \nu} & i \tilde{\mathcal{\tau}} \\
\hline \mathrm{a} & -\frac{3}{8} \Delta_{\epsilon}-\frac{3}{4}+\frac{\sqrt{3}}{8} \pi & -\frac{3}{8} \Delta_{\epsilon}-\frac{3}{4}+\frac{\sqrt{3}}{8} \pi \\
\mathrm{b} & -\frac{3}{8} \Delta_{\epsilon}-\frac{3}{4}+i \pi\left(-\frac{3}{8}\right) & -\frac{5}{8} \Delta_{\epsilon}-\frac{5}{4}+i \pi\left(-\frac{5}{8}\right) \\
\mathrm{c} & 2\left[-\frac{1}{4} \Delta_{\epsilon}-\frac{1}{4}\right] \\
\mathrm{d} & \frac{3}{8} \Delta_{\epsilon}+\frac{3}{2}-\frac{3 \sqrt{3}}{8} \pi+\frac{\pi^{2}}{12} & \frac{\pi^{2}}{12} \\
\mathrm{e} & \frac{1}{8} \Delta_{\epsilon}+\frac{\pi^{2}}{48}+i \pi\left(\frac{1}{4} \ln 2-\frac{1}{8}\right) & \frac{\pi^{2}}{48}+i \pi\left(\frac{1}{4} \ln 2\right) \\
\mathrm{f} & 2\left[\frac{1}{8} \Delta_{\epsilon}+\frac{1}{4}+i \pi\left(\frac{1}{8}\right)\right] & \\
\mathrm{ct} & -\frac{1}{8} & \frac{3}{2} \Delta_{\epsilon}+\frac{23}{8}-\frac{3 \sqrt{3}}{8} \pi
\end{array}
$$

Table 2: The one-loop vertex contributions in the Standard Model . Each entry is multiplied by common factor $\frac{1}{2} \frac{g^{3}}{16 \pi^{2}} \frac{m^{4}}{M^{3}}$

and neutral Goldstone bosons, there are two additional(equal) triangle diagrams for $h \rightarrow W_{+} W_{-}$in which all three internal lines are Goldstone boson propagators. We denote these diagrams 2.f. The evaluation of these Feynman diagrams follows from the Feynman rules deduced from the Lagrangian (64). The results are given in Table 2. To evaluate the contributions from the counter terms (fig.4), we require the counter terms from [5]. Since there is no dynamics from the gauge sector in the Lagrangian (64), $\delta Z_{W}=0, \quad \delta Z_{g}=0$. Then, $Z_{4 g}=Z Z_{W} Z_{g}=Z$. In [5], just as in the gaugeless limit of the Abelian Higgs model considered above, also $\delta \zeta_{v}=0$ and $Z=Z_{\phi}\left(=Z_{\phi}^{\star}\right)$. So the counter terms are $\left(Z_{4 \lambda}=Z^{2} Z_{\lambda}\right)$

$$
\begin{gathered}
\frac{g}{2} \frac{m^{2}}{M} \delta Z=\frac{g}{2} \frac{m^{2}}{M} \frac{g^{2}}{16 \pi^{2}} \frac{m^{2}}{M^{2}}\left[-\frac{1}{8}\right] \\
\frac{g}{2} \frac{m^{2}}{M} \delta Z_{4 \lambda}=\frac{g}{2} \frac{m^{2}}{M} \frac{g^{2}}{16 \pi^{2}} \frac{m^{2}}{M^{2}}\left[\frac{3}{2} \Delta_{\epsilon}+\frac{23}{8}-\frac{3 \sqrt{3}}{8} \pi\right]
\end{gathered}
$$

which appear as the last entries in Table2.

For the external line factors there is no contribution from $Z=Z^{\star}$, but we need

$$
\delta Z_{h}^{\star}=\frac{g^{2}}{16 \pi^{2}} \frac{m^{2}}{M^{2}}\left[\frac{3}{2}-\frac{\sqrt{3}}{4} \pi\right]
$$

So the external line factor for both processes is

$$
\sqrt{\frac{Z_{h}^{\star}}{Z}}=1+\frac{1}{2}\left(\delta Z_{h}^{\star}-\delta Z\right)=1+\frac{g^{2}}{16 \pi^{2}} \frac{m^{2}}{M^{2}}\left[\frac{13}{16}-\frac{\sqrt{3}}{8} \pi\right]
$$

Combining all of these,including the imaginary part from (67) gives the result

$$
\frac{k_{2}^{\nu} k_{1}^{\mu}}{M^{2}} \mathcal{M}_{\mu \nu}=\frac{g}{2} \frac{m^{2}}{M}\left\{1+\frac{g^{2}}{16 \pi^{2}} \frac{m^{2}}{M^{2}}\left[\frac{19}{16}-\frac{3 \sqrt{3}}{8} \pi+\frac{5}{48} \pi^{2}+\frac{i \pi}{4}\left(\ln 2-\frac{5}{2}\right)\right]\right\}=-\mathcal{M}
$$

The real part agrees with old one-loop calculations [8] of both amplitudes . Recent two-loop calculations [9] based on the Equivalence Theorem have obtained the imaginary part of $-\mathcal{M}$ in agreement with (73). 


\section{Discussion}

The working out of the Equivalence Theorem in this example is rather remarkable. It provides further testimony to the importance of the complex pole mass of the unstable particle (see also [7]). This author's original thought was that treatment of the external Higgs boson mass could not affect the Equivalence Theorem because it would be common to all the processes related by the Equivalence Theorem ; but in some cases $m^{2}$ appearing in the tree amplitude is a renormalized Lagangian parameter with no imaginary part, and in other cases it is the square of the Higgs boson fourmomentum which does have an imaginary part.

The calculation is also consistent with the general principle of renormalized perturbation theory that analyticity and unitarity require real renormalization constants. Renormalization only provides subtractions for the real parts of self-energy functions. A problem which will have to be faced in higher order is the different real renormalized masses $m_{r}^{2}$ and $m_{p}^{2}$ discussed below (47) [7].

\section{A Renormalization constants and Vev in the Abelian Higgs model}

The renormalization constants and vev which satisfy the five specified conditions are calculated in the leading order $g^{2} \frac{m^{2}}{M^{2}}$, which remains finite in the limit considered. (It is convenient to calculate the scalar vertex renormalization constant in place of $Z_{\lambda}$ )

$$
\begin{gathered}
\delta \zeta_{v}=0 \\
\delta Z_{A}=0 \\
\delta Z=\frac{g^{2}}{16 \pi^{2}} \frac{m^{2}}{M^{2}}\left[-\frac{1}{2}\right] \\
\delta Z_{\mu^{2}}=\frac{g^{2}}{16 \pi^{2}} \frac{m^{2}}{M^{2}}\left[2 \Delta_{\epsilon}+7-\frac{3 \sqrt{3}}{2} \pi\right] \\
\delta Z_{4 \lambda}=\frac{g^{2}}{16 \pi^{2}} \frac{m^{2}}{M^{2}}\left[5 \Delta_{\epsilon}+\frac{19}{2}-\frac{3 \sqrt{3}}{2} \pi\right]
\end{gathered}
$$

In these formulas $\Delta_{\epsilon}$ is the usual dimensional regularization quantity $\frac{2}{4-d}-\gamma_{E}+\ln 4 \pi$

For use in $(\sqrt{72})$, we also need the star-Z's

$$
\begin{gathered}
\delta Z_{h}^{\star}=\frac{g^{2}}{16 \pi^{2}} \frac{m^{2}}{M^{2}}[5-\sqrt{3} \pi] \\
\delta Z_{\chi}^{\star}=\frac{g^{2}}{16 \pi^{2}} \frac{m^{2}}{M^{2}}\left[-\frac{1}{2}\right]
\end{gathered}
$$




\section{B Acknowledgements}

I particularly thank an anonymous referee for his/her hunch that the Equivalence Theorem shuld work out if the decay amplitude of the unstable Higgs boson is evaluated at its complex on-shell value.

I thank Y.-Y.Charng for checking some of the calculations.

\section{References}

[1] H.-J.He,Y.-P.Kuang, and C.-P.Yuan,hep-ph/9704276. H.-J.He and W.B.Kilgore, hep-ph/9609320.

[2] Textbook treatments of BRST symmetry may be found in M.E.Peskin and D.V.Schroeder, An Introduction to Quantum Field Theory Addison- Wesley,1995, and S.Weinberg, The Quantum Theory of Fields, Volume II Cambridge, 1996 .

[3] R.J.Eden,P.V.Landshoaff,D.I.Olive,J.C.Polkinghorne, The Analytic S-Matrix, Cambridge University Press (1966)

[4] H.Veltman,Phys. Rev. D41 2294 (1990)

[5] A.I.Bochkarev and R.S.Willey,hep-ph/9607240,

[6] M.Bohm,H.Speisberger,and W.Hollik,Fort.Phys.38 165(1990).

W.Hollik,Fort.Phys.34687(1986);

[7] R.G.Stuart,hep-ph/9706550; M.Passera and A.Sirlin, hep-ph/9804309; B.A.Kniehl and A.Sirlin,hep-ph/9805390.

[8] J.Fleischer and F.Jegerlehner,Phys. Rev. D23 2001 (1981)for $h \rightarrow W W$ and W.J.Marciano and S.S.D.Willenbrock,Phys. Rev. D37 2509 (1988) for $h \rightarrow \phi \phi$

[9] A.Ghinculov,Nucl. Phys. B455 21 (1995),hep-ph/9507240; A.frink,B.A.Kniehl, D.Kreimer,and K.Riesselmann,Phys. Rev. D54 4548 (1996). 